\title{
Electrical compensation via vacancy-donor complexes in arsenic-implanted and laser-annealed germanium
}

\author{
T. Kalliovaara, ${ }^{1, a)}$ J. Slotte,${ }^{1}$ I. Makkonen, ${ }^{1}$ J. Kujala, ${ }^{1}$ F. Tuomisto, ${ }^{1}$ R. Milazzo, ${ }^{2}$ \\ G. Impellizzeri, ${ }^{3}$ G. Fortunato, ${ }^{4}$ and E. Napolitani ${ }^{2}$ \\ ${ }^{1}$ Department of Applied Physics, Aalto University, P.O. Box 15100, FIN-00076 Aalto, Espoo, Finland \\ ${ }^{2}$ Dipartimento di Fisica e Astronomia and CNR-IMM MATIS, Università di Padova, Via Marzolo 8 , \\ 35131 Padova, Italy \\ ${ }^{3}$ CNR-IMM, Via S. Sofia 64, 95123 Catania, Italy \\ ${ }^{4}$ CNR-IMM, Via del Fosso del Cavaliere 100, 00133 Roma, Italy
}

(Received 2 September 2016; accepted 16 October 2016; published online 1 November 2016)

\begin{abstract}
Highly $n$-type Ge attained by shallow As implantation and excimer laser annealing was studied with positron annihilation spectroscopy and theoretical calculations. We conclude that a high concentration of vacancy-arsenic complexes was introduced by the doping method, while no sign of vacancies was seen in the un-implanted laser-annealed samples. The arsenic bound to the complexes contributes substantially to the passivation of the dopants. Published by AIP Publishing.

[http://dx.doi.org/10.1063/1.4966947]
\end{abstract}

Research on Ge has seen a revival during the past decade, after years of neglect due to the premier role of $\mathrm{Si}$ in the electronics industry. Particular efforts have been made to deploy the superior charge carrier mobility of Ge in complementary metal-oxide-semiconductor usage. ${ }^{1} \mathrm{~A}$ few hindrances exist, however, including the poor quality of the native surface oxide $\mathrm{GeO}_{2}{ }^{2}$ and the difficulty in obtaining high-enough $n$-type carrier concentrations for ultra-shallow junctions. ${ }^{3}$ While the insulation issue has essentially been resolved by introducing high- $\kappa$ dielectrics as a replacement for $\mathrm{SiO}_{2}$ a decade ago (directly applicable to $\mathrm{Ge}$ ), the doping limitations are still to be overcome. Hence, it is imperative to have more profound knowledge on the formation of defects and their role in the carrier compensation of Ge.

The difficulty in achieving high $n$-type doping levels arises from the lower solubility and higher diffusivity of group- $\mathrm{V}$ atoms in $\mathrm{Ge} .^{5}$ The latter has been associated with a vacancy-mediated diffusion mechanism, ${ }^{6}$ as opposed to $\mathrm{Si}$, wherein donors diffuse through both vacancies ( $V$ 's) and interstitials. ${ }^{7}$ Furthermore, donor passivation is known to be a problem in $\mathrm{Ge}$, and it has been attributed to the formation of vacancy-donor $\left(V_{M}-D_{N}\right)$ complexes in a few experimen$\mathrm{tal}^{8-11}$ and computational ${ }^{12}$ studies. In a recent positron study on highly $n$-type Ge obtained by diffusion doping, an extensive formation of $V-D_{N}$ complexes was found with the common donors $D \in\{\mathrm{As}, \mathrm{P}, \mathrm{Sb}\}{ }^{13}$

In this paper, we apply positron annihilation spectroscopy to study the defects in Ge heavily doped with As. The measured samples were (100)-oriented Czochralski-grown Ge crystals (initially lightly $n$-type, $\rho>40 \Omega \mathrm{cm}$ ), implanted with $40 \mathrm{keV} \mathrm{As}{ }^{+}$ions with a fluence of either $2 \times 10^{14}$ or $3 \times 10^{15} \mathrm{~cm}^{-2}$, hereafter labeled As1 and As2. The implantation produced an amorphous layer of 48 and $61 \mathrm{~nm}$ for the low and high fluence, respectively, in good agreement with the critical damage energy density model. ${ }^{14}$ Subsequently,

a)tuomas.kalliovaara@aalto.fi the samples were subject to melting laser thermal annealing (LTA), using excimer laser $(\lambda=308 \mathrm{~nm})$ pulses of $28 \mathrm{~ns}$ with an energy density of either 530 or $650 \mathrm{~mJ} / \mathrm{cm}^{2}$ (labeled $\mathrm{L} x$ and $\mathrm{H} x$, for $x$ pulses of low and high energy density, respectively), which recrystallized the amorphized layer. The enhanced diffusivity of the dopants in the liquid phase resulted in a roughly homogeneous As profile down to the melt depth (55-120 nm depending on the energy density), with a maximum As concentration below and above the equilibrium solubility limit of $8 \times 10^{19} \mathrm{~cm}^{-3}$ (Ref. 5) for As1 and As2, respectively. One of the samples (As1 L5) was annealed repetitively by five pulses with a rate of $\sim 1 \mathrm{~Hz}$. The As1 samples attained a total As concentration of $\sim 4$ $\times 10^{19} \mathrm{~cm}^{-3}$, out of which $\sim 2 \times 10^{19} \mathrm{~cm}^{-3}$ was active. For As2 L1, the corresponding figures were $\sim 5 \times 10^{20} \mathrm{~cm}^{-3}$ and $\sim 8 \times 10^{19} \mathrm{~cm}^{-3}$. In addition, two virgin (v-Ge) samples were irradiated with single low- and high-energy pulses, respectively, providing reference of the effect of the laser alone. For a comprehensive description of the preparation and characterizations of the samples, the reader can refer to Ref. 15. In brief, secondary ion mass spectrometry (SIMS) and spreading resistance profiling revealed As activation no better than $50 \%$, which motivated the present study.

All the samples were measured with a variable-energy positron beam, using a single high-purity Ge detector with a resolution of $1.08 \mathrm{keV}$ at $511 \mathrm{keV}$. The annihilation spectra were characterized by the conventional shape parameters $S$ and $W$, with the integration windows set to $|p|<0.41$ a.u. for $S$ and 1.6 a.u. $<|p|<4.0$ a.u. for $W, p$ denoting the Doppler shift in terms of momentum. For a bulk Ge reference, we determined the values of $S_{\mathrm{Ge}}=0.540$ and $W_{\mathrm{Ge}}=0.0321$, to which the parameters of the samples were scaled. Two of the samples were inspected more closely with a coincidence-Doppler setup, which encompasses an improved peak-to-background ratio. One of the collinear high-purity Ge detectors was used to impose the time-coincidence condition on the pulses recorded by the other with a resolution of $1.20 \mathrm{keV}$. A detailed description of the experimental technique is given in Ref. 16. 
Fig. 1 reports the $S$ parameters measured throughout the energy range. As seen in the figure, the two un-implanted samples (v-Ge L1 and v-Ge H1) do not show the signs of vacancies: their relative $S$ parameters increase slowly as a function of $E$, corresponding to a large positron diffusion length, and settle both to unity eventually. In contrast, all the doped samples exhibit a steep increase in $S$, indicating significantly suppressed positron diffusion due to trapping. The $S$ parameters peak to $4.5 \%-5.5 \%$ above the bulk value, signifying that the traps are larger in open volume than a monovacancy. ${ }^{13}$ The fact that no vacancies are detected due to LTA alone is quite surprising; in particular, it is different from $\mathrm{Si}$ wherein $V$ generation and clustering caused by pulsed laser irradiation has been observed. ${ }^{17}$ Milazzo et al.${ }^{18}$ arrived at the same conclusion based on the changes from the oxygeninduced strain measured in virgin Ge and attributed to interstitial occupation. In their study, the doped samples showed negligible $\mathrm{O}$ contribution to the strain, which was associated with vacancy-oxygen complexes, while $\mathrm{O}$ itself was found uncorrelated with the As passivation. ${ }^{19}$ Hence, As appears to play a key role in $V$ generation even during the strongly nonequilibrium recrystallization.

The difference between the results of un-implanted $\mathrm{Si}$ and Ge might arise from the clear acceptor nature of the vacancy in $\mathrm{Ge}$ (Ref. 20) in contrast to $\mathrm{Si}$, where the acceptor levels of $V$ lie closer to the conduction band. ${ }^{21}$ Consequently, a substantial fraction of $V$ 's might occur in the neutral charge state in lightly $n$-type $\mathrm{Si}$, allowing them to pair into divacancies. On the contrary, the negatively charged $V$ 's in Ge repel each other and anneal out unless enough donors are present to pair with. Milazzo et al., ${ }^{18}$ however, suggested that $V$ production in the liquid-phase epitaxy of Ge would be very limited. Instead, a vast amount of $V$-As pairs would be generated at the liquid-solid interface, and their dissociation into $V$ and substitutional As $\left(\mathrm{As}_{\mathrm{Ge}}\right)$ would give rise to a supersaturation of $V$ 's within the regrown layer. Similar to $V, V$-As is also

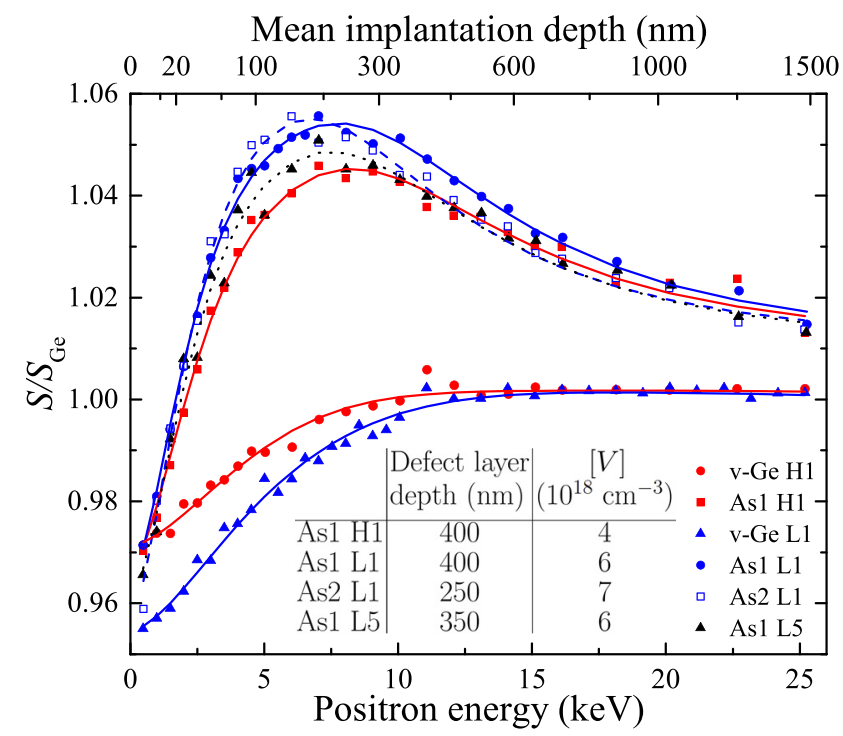

FIG. 1. $S(E)$ plots for all the samples scaled to the bulk-Ge reference. The mean positron implantation depth is indicated on the top axis. The line plots are fits to the $S$-parameter profiles performed with the VEPFIT program, which yielded the estimates reported in the inset. No vacancies are detected in the un-implanted samples. highly diffusive ${ }^{9}$ and will readily react with $\mathrm{As}_{\mathrm{Ge}}$ during the migration, producing stable $V-\mathrm{As}_{2}$ clusters. ${ }^{22}$ Additional encounters of $V$ 's or $V$-As's with such defects would then result in larger complexes.

In Fig. 2, we present the shape parameters of the doped samples in the $S-W$ plane, again scaled to the bulk values. The measurement data of each sample lie roughly on two separate line segments, corresponding to superpositions between the surface and a trap state and between the trap and the bulk state, respectively. The turning between the line segments occurs sharply, indicating saturation trapping, i.e., effectively all positrons annihilate trapped in the same kind of a defect. Thus, the trap-related parameters point toward

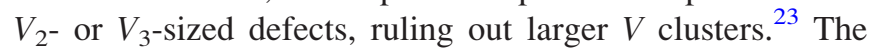
differences in the turning point between the four samples appear relatively small, which implies that the trapping defects could be of the same kind in all of the samples. Saturation trapping of positrons also explains why the data are similar for different As contents.

In order to estimate the defect concentration, the stationary positron diffusion equation was fitted with VEPFIT $^{24}$ to the $S$-parameter data. We applied a simple model with a homogeneous defect layer on top of a Ge substrate and fitted the layer thickness and the effective positron diffusion length $(30 \ldots 40 \mathrm{~nm})$ in the layer for each doped sample. The latter were used to estimate the average vacancy concentration $([V])$ as in Ref. 25. The obtained estimates are given in the inset of Fig. 1. We stress that the values are crudely approximative and should only be considered as orders of magnitude, e.g., $[V]=10^{18} \ldots 10^{19} \mathrm{~cm}^{-3}$. Assuming that the vacancies are decorated by a few As atoms, such concentrations seem plausible to account for the carrier compensation - particularly for the low-fluence (As1) samples, where $[V]$ is comparable with the concentration of passive As $\left(\sim 10^{19} \mathrm{~cm}^{-3}\right)$. In the highfluence sample (As2 L1), $[V]$ was estimated to be only slightly higher, even though the sample contains approximately an order of magnitude more of passive As $\left(\sim 10^{20} \mathrm{~cm}^{-3}\right)$. However, as the vacancy concentration exceeds the positron saturation trapping concentration, the fitting becomes

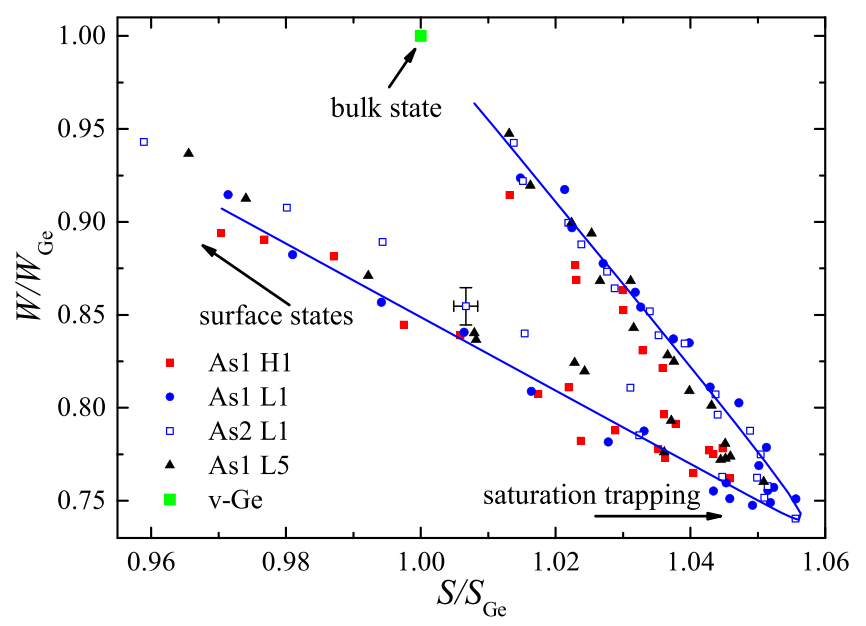

FIG. 2. The line-shape parameters of the doped samples presented in the parameter plane. Three distinct annihilation states can be observed, as indicated by the arrows. Typical error bars due to the Poissonian nature of the annihilation events are shown for the data point in the middle of the figure. A VEPFIT fit for As1 L1 is included. 
increasingly insensitive to changes in the effective positron diffusion length. Hence, it is likely that vacancy-arsenic complexes cause the passivation also in As2 L1.

From the VEPFIT analysis (and the $S$-parameter values peaking at mean implantation depths of roughly $200 \mathrm{~nm}$; see Fig. 1), it is evident that the vacancy distribution extends markedly beyond the melt depth in each of the doped samples. This is rather a peculiar observation, as the SIMS profiles ${ }^{15}$ show that the As concentration drops abruptly at depths exceeding the melted layer. Various implantation studies on $\mathrm{Si}$ and $\mathrm{SiC}$ have found the vacancy-type defects well beyond the projected range of the implanted ions, and it has often been suggested to be due to vacancies produced within the projected range diffusing deeper into the sample. ${ }^{26}$ In a study on $4 \mathrm{H}-\mathrm{SiC}$, it was shown, however, that ion channeling can have a substantial impact and potentially explains many of the observations attributed to vacancy migration. ${ }^{27}$ Extrapolating the SIMS data ${ }^{15}$ for the exponentially decaying channeling tail, it can be concluded that the As concentration falls slightly below $10^{16}$ and $10^{17} \mathrm{~cm}^{-3}$ at $200 \mathrm{~nm}$ for As1 and As2, respectively, dropping by roughly two orders of magnitude more at $300 \mathrm{~nm}$. It seems unlikely that such low implant concentrations could account for the detectable amounts of $V$-type defects below the regrown layer. Further investigations (out of the scope of this work) are required in order to identify the origin and detailed nature of the vacancy defects in this region.

In order to identify the defects introduced by the implantation-annealing processing, we measured the two samples (As1 L1 and As2 L1), wherein positron trapping appeared strongest with the coincidence setup. Fig. 3(a) reports the results in the form of ratio curves, i.e., the annihilation intensity is given relative to that measured for a bulk sample. In addition, we show a curve from Ref. 13, identified as a characteristic of $V$-As complexes with $N \geq 3$, from which the results obtained in this study differ remarkably. In particular, the annihilation intensity at low (high) momenta of the electron-positron pairs is significantly higher (lower) for the present samples, confirming the above conclusion that the defects are larger in open volume than a monovacancy. Furthermore, the $V$-As $s_{N}$ curve displays a prominent shoulder (or a blunt peak) at $\sim 1.2$ a.u., but in the implanted samples it barely exists. In order to see whether there would be a difference between the positron-trapping defects in the melted layer and those below it, we measured the samples with the positron energies of 5 and $10 \mathrm{keV}$, corresponding to the mean implantation depths of approximately 100 and $300 \mathrm{~nm}$, respectively. Indeed, both As1 L1 and As2 L1 measured at $E=5 \mathrm{keV}$ show a higher intensity in the momentum region between 1.0 and 1.5 a.u., and a lower intensity from 1.8 a.u. onward, compared to the $10 \mathrm{keV}$ spectra. In order to rule out the possibility for the difference being due to annihilation at the surface, a spectrum was measured also at $E=1 \mathrm{keV}$ for As1 L1. While the corresponding ratio curve exhibits a high peak in the shoulder region, it seems unlikely to explain the pronounced shoulder in the $5 \mathrm{keV}$ curve, since at $E=1 \mathrm{keV}$ the intensity is very low in the low-momentum regime and substantially higher at and around 2 a.u. compared to the $5 \mathrm{keV}$ spectrum.

In order to interpret the coincidence spectra, we modeled a few vacancy-arsenic complexes based on the twocomponent density-functional theory (DFT), employing the

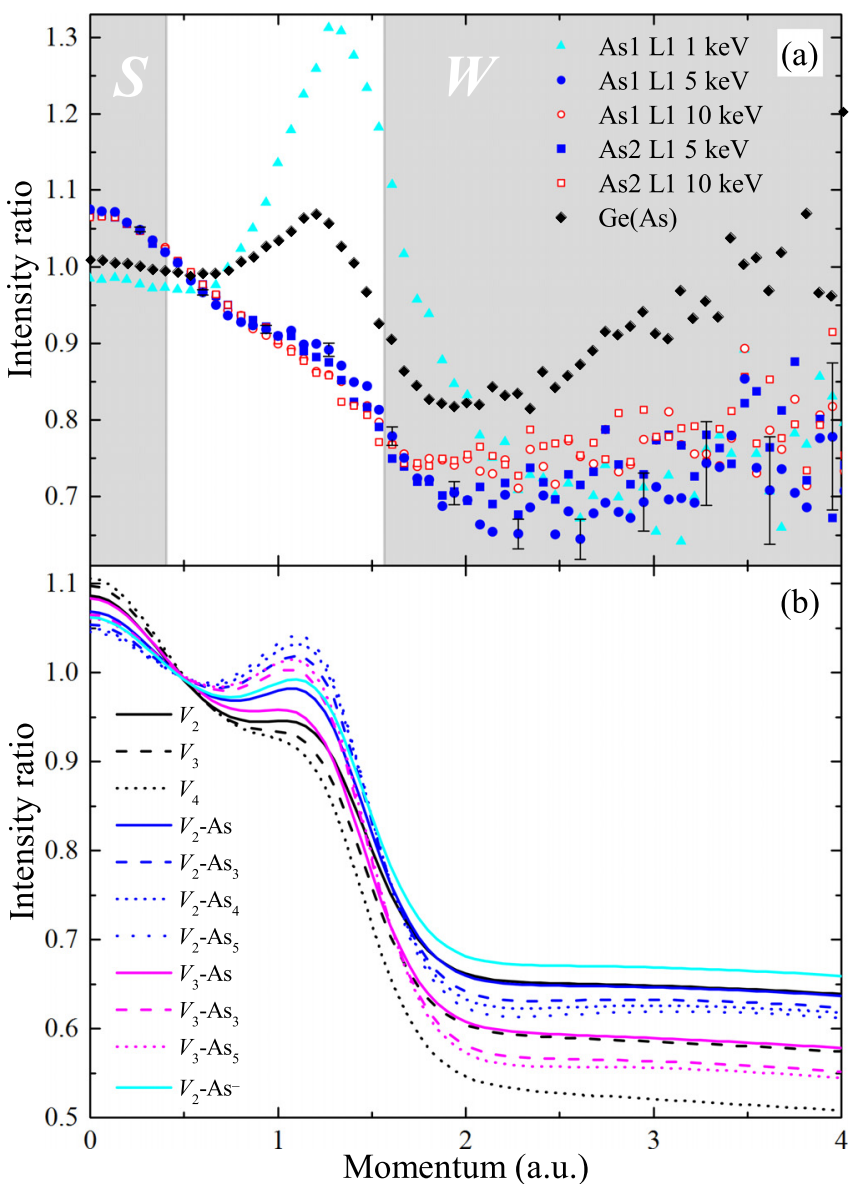

FIG. 3. (a) Annihilation intensities (scaled to that of pure Ge) measured for the samples As1 L1 and As2 L1. For comparison, a ratio curve [Ge(As)] from a previous study ${ }^{13}$ (that used the same reference sample) is presented. A few error bars have been plotted to show how the uncertainty in the data increases at higher momenta due to scarcity of counts. The $S$ and $W$ windows have also been shaded. (b) Annihilation-intensity ratios computed for various vacancy-arsenic complexes in Ge. Each spectrum (including the bulk reference) was integrated in the (100) crystallographic direction; the differences between different directions were minor. The spectra were convoluted with the experimental resolution function.

plane-wave code VASP. ${ }^{28}$ A description of our computational scheme can be found in, e.g., Ref. 13 and the references therein. We calculated the ratio curves for $V_{2}$ and $V_{3}$ decorated by the 1-5 nearest-neighbor As atoms, and for pure vacancy clusters $\left(V_{2} \ldots V_{4}\right)$, in the neutral charge state. In addition, we studied a singly negative $V_{2}$-As complex. The problem with the underestimated band gap due to using the local-density approximation was circumvented by sampling the Brillouin zone at the corner point, where the gap remains finite. ${ }^{29}$ The results are reported in Fig. 3(b). In terms of adding As atoms to the complex, a clear trend can be observed: The more As, the higher a shoulder appears at $\sim 1.2$ a.u., resulting from the additional valence electron of As and an inward relaxation of the defect. Simultaneously, the intensity is decreased at low and high momenta. If the complex is negatively charged, inward relaxation is promoted and the shoulder further increased.

Compared to the measured intensity ratios of Fig. 3(a), the calculations suggest a very prominent shoulder for the $V_{2}-\mathrm{As}_{N}$ complexes. While an additional $V$ reduces the shoulder, the minor hump in the experimental curves is not 
recovered. In fact, even the pure tetravacancy $\left(V_{4}\right)$ displays more shoulder than the measured curves. Nevertheless, complexes comprising more than $3 \mathrm{~V}$ 's cannot explain the data, as their larger open volume results in too high (low) intensities at low (high) momenta. Therefore, we interpret the measured ratio curves such that the dominant defects found in the regrown layer are di- or trivacancies decorated by As atoms, and the defects beyond the layer are equal in open volume but neighbored by fewer (if any) As atoms. Considering the diffusion mechanism resulting in the formation of the defects, the $V_{2}-\mathrm{As}_{N}$ complexes seem more likely to form.

In summary, we have applied positron annihilation spectroscopy and DFT calculations to investigate the openvolume defects formed through shallow As implantation at high fluences and subsequent melting laser annealing in Ge. While the virgin Ge samples showed no signs of vacancy generation after single laser pulses, a high concentration $\left(10^{18} \ldots 10^{19} \mathrm{~cm}^{-3}\right)$ of vacancy-arsenic complexes larger in open volume than a monovacancy was observed in the doped samples. Interestingly, the vacancy distribution considerably exceeded the melt depth, and the deep-lying defects were found to contain fewer As atoms than those within the regrown layer. The vacancy-arsenic complexes contribute substantially to the large inactive fraction of the donors.

We acknowledge the computational resources provided by the Aalto Science-IT project and CSC - IT Center for Science, Finland. T.K. and I.M. acknowledge the financial support from the Academy of Finland (Project Nos. 285809 and 293932).

${ }^{1}$ M. L. Lee, E. A. Fitzgerald, M. T. Bulsara, M. T. Currie, and A. Lochtefeld, J. Appl. Phys. 97, 011101 (2005).

${ }^{2}$ K. Prabhakaran and T. Ogino, Surf. Sci. 325, 263 (1995).

${ }^{3}$ E. Simoen, A. Satta, A. D'Amore, T. Janssens, T. Clarysse, K. Martens, B. De Jaeger, A. Benedetti, I. Hoflijk, B. Brijs et al., Mater. Sci. Semicond. Proc. 9, 634 (2006).

${ }^{4}$ K. Mistry, C. Allen, C. Auth, B. Beattie, D. Bergstrom, M. Bost, M. Brazier, M. Buehler, A. Cappellani, R. Chau et al., in Proceedings of the IEEE International Electron Devices Meeting (2007), pp. 247-250.

${ }^{5}$ Germanium-Based Technologies: From Materials to Devices, edited by C. Claeys and E. Simoen (Elsevier, 2011).

${ }^{6}$ M. Werner, H. Mehrer, and H. D. Hochheimer, Phys. Rev. B 32, 3930 (1985).

${ }^{7}$ H. Bracht, H. H. Silvestri, I. D. Sharp, and E. E. Haller, Phys. Rev. B 75, 035211 (2007).

${ }^{8}$ A. R. Peaker, V. P. Markevich, B. Hamilton, I. D. Hawkins, J. Slotte, K. Kuitunen, F. Tuomisto, A. Satta, E. Simoen, and N. V. Abrosimov, Thin Solid Films 517, 152 (2008).
${ }^{9}$ S. Brotzmann and H. Bracht, J. Appl. Phys. 103, 033508 (2008).

${ }^{10}$ S. Brotzmann, H. Bracht, J. L. Hansen, A. N. Larsen, E. Simoen, E. E. Haller, J. S. Christensen, and P. Werner, Phys. Rev. B 77, 235207 (2008).

${ }^{11}$ G. Impellizzeri, S. Boninelli, F. Priolo, E. Napolitani, C. Spinella, A. Chroneos, and H. Bracht, J. Appl. Phys. 109, 113527 (2011).

${ }^{12}$ J. Coutinho, V. J. B. Torres, A. Carvalho, R. Jones, S. Öberg, and P. R. Briddon, Mater. Sci. Semicond. Proc. 9, 477 (2006); J. Coutinho, V. J. B. Torres, S. Öberg, A. Carvalho, C. Janke, R. Jones, and P. R. Briddon, J. Mater. Sci. Mater. Electron. 18, 769 (2007); J. Coutinho, C. Janke, A. Carvalho, V. J. B. Torres, S. Öberg, R. Jones, and P. R. Briddon, Physica B 401, 179 (2007).

${ }^{13}$ J. Kujala, T. Südkamp, J. Slotte, I. Makkonen, F. Tuomisto, and H. Bracht, J. Phys. Condens. Matter 28, 335801 (2016).

${ }^{14}$ G. Impellizzeri, S. Mirabella, and M. G. Grimaldi, Appl. Phys. A 103, 323 (2011).

${ }^{15}$ R. Milazzo, E. Napolitani, G. Impellizzeri, G. Fisicaro, S. Boninelli, M. Cuscunà, D. De Salvador, M. Mastromatteo, M. Italia, A. La Magna et al., J. Appl. Phys. 115, 053501 (2014).

${ }^{16}$ F. Tuomisto and I. Makkonen, Rev. Mod. Phys. 85, 1583 (2013).

${ }^{17}$ A. La Magna, V. Privitera, G. Fortunato, M. Cuscunà, B. G. Svensson, E. Monakhov, K. Kuitunen, J. Slotte, and F. Tuomisto, Phys. Rev. B 75, 235201 (2007).

${ }^{18}$ R. Milazzo, G. Impellizzeri, D. Piccinotti, A. La Magna, G. Fortunato, D. De Salvador, A. Carnera, A. Portavoce, D. Mangelinck, V. Privitera, and E. Napolitani, J. Appl. Phys. 119, 045702 (2016).

${ }^{19}$ R. Milazzo, G. Impellizzeri, M. Cuscunà, D. D. Salvador, M. Mastromatteo, A. L. Magna, G. Fortunato, F. Priolo, V. Privitera, A. Carnera, and E. Napolitani, Mater. Sci. Semicond. Proc. 42, 196 (2016).

${ }^{20}$ J. Coutinho, R. Jones, V. J. B. Torres, M. Barroso, S. Öberg, and P. R. Briddon, J. Phys. Condens. Matter 17, L521 (2005).

${ }^{21}$ E. G. Seebauer and M. C. Kratzer, Charged Semiconductor Defects: Structure, Thermodynamics and Diffusion (Springer Science and Business Media, 2008).

${ }^{22}$ A. Chroneos, R. W. Grimes, B. P. Uberuaga, S. Brotzmann, and H. Bracht, Appl. Phys. Lett. 91, 192106 (2007).

${ }^{23}$ J. Slotte, M. Rummukainen, F. Tuomisto, V. P. Markevich, A. R. Peaker, C. Jeynes, and R. M. Gwilliam, Phys. Rev. B 78, 085202 (2008).

${ }^{24}$ A. Van Veen, H. Schut, J. De Vries, R. A. Hakvoort, and M. R. Ijpma, AIP Conf. Proc. 218, 171 (1991).

${ }^{25}$ S. Eichler, J. Gebauer, F. Börner, A. Polity, R. Krause-Rehberg, E. Wendler, B. Weber, W. Wesch, and H. Börner, Phys. Rev. B 56, 1393 (1997).

${ }^{26}$ A. Uedono, L. Wei, C. Dosho, H. Kondo, S. Tanigawa, J. Sugiura, and M. Ogasawara, Jpn. J. Appl. Phys., Part 1 30, 201 (1991); A. Uedono, L. Wei, C. Dosho, H. Kondo, S. Tanigawa, and M. Tamura, Jpn. J. Appl. Phys., Part 1 30, 1597 (1991); M. Fujinami and N. B. Chilton, J. Appl. Phys. 73, 3242 (1993); Y. Pacaud, J. Stoemenos, G. Brauer, R. A. Yankov, V. Heera, M. Voelskow, R. Kögler, and W. Skorupa, Nucl. Instrum. Methods B 120, 177 (1996).

${ }^{27}$ M. S. Janson, J. Slotte, A. Y. Kuznetsov, K. Saarinen, and A. Hallén, J. Appl. Phys. 95, 57 (2004).

${ }^{28}$ G. Kresse and J. Furthmüller, Comput. Mater. Sci. 6, 15 (1996); G. Kresse and J. Furthmüller, Phys. Rev. B 54, 11169 (1996); G. Kresse and D. Joubert, Phys. Rev. B 59, 1758 (1999); I. Makkonen, M. Hakala, and M. J. Puska, J. Phys. Chem. Solids 66, 1128 (2005); Phys. Rev. B 73, 035103 (2006).

${ }^{29}$ H. M. Pinto, J. Coutinho, V. J. B. Torres, S. Öberg, and P. R. Briddon, Mater. Sci. Semicond. Proc. 9, 498 (2006). 